\title{
The Effelsberg $21 \mathrm{~cm}$ radio continuum survey of the Galactic plane between $l=95.5$ and $l=240^{\circ}$ *
}

\author{
P. Reich, W. Reich, and E. Fürst \\ Max-Planck-Institut für Radioastronomie, Auf dem Hügel 69, D-53121 Bonn, Germany \\ Received March 4; accepted April 11, 1997
}

\begin{abstract}
We present the results of a radio continuum survey at $21 \mathrm{~cm}$ wavelength of the Galactic plane between $95.5 \leq l \leq 240^{\circ}$ and $-4^{\circ} \leq b \leq+5^{\circ}$ with the Effelsberg 100-m telescope. The data have been absolutely calibrated by using the Stockert northern sky survey at the same wavelength. This survey complements the Effelsberg $21 \mathrm{~cm}$ Galactic plane survey for $l \leq 95^{\circ} .5$ by Reich et al. (1990a). The results are presented as contour maps with a sensitivity of $100 \mathrm{mK} T_{B}$ at an angular resolution (HPBW) of 9!4. In addition we extend the list of compact sources given by Reich et al. (1990a) by 1830 new entries. These sources have peak flux densities exceeding $160 \mathrm{mK} T_{B}$ (or $79 \mathrm{mJy} /$ beam area) and apparent sizes of less than $16^{\prime}$.
\end{abstract}

Key words: catalogs — surveys — galactic: general

\section{Introduction}

Reich et al. (1990a, Paper I) have presented the Effelsberg $21 \mathrm{~cm}$ radio continuum survey of the Galactic plane for longitudes between $l=357^{\circ}$ and $l=95.5$ and latitudes $-4^{\circ} \leq b \leq+4^{\circ}$. Here we complement these maps for the Galactic plane region towards the anticentre direction up to $l=240^{\circ}$. The latitude extent is slightly enlarged for some regions to $b=+5^{\circ}$. The area from $93^{\circ} \leq l \leq 162^{\circ}$, $-4^{\circ} \leq b \leq+4^{\circ}$ has been previously surveyed for continuum radiation at $21 \mathrm{~cm}$ wavelength with the $100-\mathrm{m}$ telescope by Kallas \& Reich (1980). This survey, which is based on observations carried out between 1974 and 1978, is of significantly lower sensitivity if compared with the

Send offprint requests to: P. Reich

* Table 1 only available in electronic form at CDS via anonymous ftp to cdsarc.u-strasbg.fr (130.79.128.5) or via http://cdsweb.u-strasbg.fr/Abstract.html
$21 \mathrm{~cm}$ radio continuum maps presented in Paper I and therefore has been reobserved to get maps of equal sensitivity along the entire Galactic plane.

\section{Observations}

The observations started in 1982 and were finished in March 1988. However, during the reduction stage numerous areas have been found to be distorted by low level interference or by far-sidelobe contributions, which may pick up solar radio emission even at very large angular distances from the observing direction (Kalberla et al. 1980). Therefore several small areas have been subsequently reobserved between December 1990 and February 1994.

We used the same receiver as described already in Paper I (see Table 1) for the observations done until March 1988. The two channel (left- and right-hand) FET based total power receiver $\left(T_{\text {sys }}=45 \mathrm{~K}\right)$ was installed in the prime focus of the telescope (Schmidt \& Wongsowijoto 1983). For the majority of observations the centre frequency was set to $1408 \mathrm{MHz}$ or $1410 \mathrm{MHz}$, but sometimes it was necessary to change to $1430 \mathrm{MHz}$ or $1440 \mathrm{MHz}$ because of the actual interference situation. For the same reason the standard bandwidth of $20 \mathrm{MHz}$ was sometimes reduced to about $10 \mathrm{MHz}$ bandwidth. Very few observations have been made with a bandwidth of $40 \mathrm{MHz}$.

A new sensitive $L$-band receiving system based on cooled HEMT amplifiers has replaced the FET receiver in 1990 and has been used for all reobservations of distorted areas. Its properties for continuum observations have been already described by Reich et al. (1992). The new receiver is quite similar to the previous receiver except that its system temperature is reduced to about $30 \mathrm{~K}$.

The method of observation was basically the same as described in Paper I. The telescope has been moved between Galactic latitudes $-4^{\circ} \leq b \leq+4^{\circ}$ with a speed of $4^{\circ} /$ minute. However, as the strength and gradients of the diffuse Galactic emission are small in the anticentre direction if compared with the properties in the first Galactic quadrant, we have made also observations by moving the telescope in longitude direction for $l \geq 162^{\circ}$. The length 
of the longitude scans has been chosen in a way that they end in regions with weak emission. Extensions towards $+5^{\circ}$ latitude have also been observed in longitude at different scan length.

Calibration is based on 3C 286 assuming $14.4 \mathrm{Jy}$. Several secondary calibrators have been observed in addition (see Paper I). Almost all areas have been observed twice.

\section{Reduction}

The principles of the applied data reduction have been already described in Paper I. However, for maps observed in orthogonal directions we applied the program PLAIT (Emerson \& Gräve 1988), which is quite effective in reducing scanning effects. The large scale structures have been added (see Paper I for details) by using the data from the $1420 \mathrm{MHz}$ Stockert continuum survey (Reich 1982; Reich \& Reich 1986). This survey is limited to the declination range above $-19^{\circ}$ and was used therefore to correct the data for Galactic longitudes $l \leq 233^{\circ}$. For longitudes $l \geq 233^{\circ}$ corrections have been applied by scaling the $408 \mathrm{MHz}$ all-sky survey (Haslam et al. 1982) with a temperature spectral index of $\beta=2.8\left(T_{B} \sim \nu^{-\beta}\right)$. A deviation in the spectral index of $\Delta \beta=0.2$ results in a temperature change of the maximum of the background emission of about $0.4 \mathrm{~K}$.

\section{The CAS A area}

There are particular sidelobe confusion problems in the area around the exceptionally strong supernova remnant CAS A. Two maps observed at different parallactic angles have been made (Figs. 1a and 1b). We have reduced this field in a different way aiming for a maximum reduction of the disturbing sidelobes. We computed the differences between the two maps. The sources disappear, but the sidelobes show up either positive or negative because of their different positions. Clipping the sidelobe effects of the second map and subtracting the result from the first map reduces their effects substantially. Similarly, the second map is improved (Figs. 2a and 2b). Both maps are added and included in the survey maps (Figs. 3 and 4 ).

\section{Results}

The results of the survey are presented in the form of contour maps and in addition a list of compact sources is given, following the presentation of the first survey section in Paper I. As described in Paper I we have decomposed the survey maps into a "source component" and a "diffuse component". The sum of both components gives the measured absolute temperature for any position. Figure 3 shows the "source component" in Galactic coordinates with a $2^{\circ}$ grid of equatorial coordinates (Epoch 1950.0) superposed. The following contour intervals are shown:

$-0.2 \mathrm{~K}$ to $1 \mathrm{~K}$ in $100 \mathrm{mK}$ steps, labelled for $0 \mathrm{~K}, 0.3 \mathrm{~K}, 0.6 \mathrm{~K}$ and dashed below $0 \mathrm{~K}$

$1 \mathrm{~K}$ to $2 \mathrm{~K}$ in $200 \mathrm{mK}$ steps, labelled for $1 \mathrm{~K}$ and $2 \mathrm{~K}$

$2 \mathrm{~K}$ to $5 \mathrm{~K}$ in $0.5 \mathrm{~K}$ steps, labelled for $5 \mathrm{~K}$

$5 \mathrm{~K}$ to $10 \mathrm{~K}$ in $1 \mathrm{~K}$ steps, labelled for $10 \mathrm{~K}$

$10 \mathrm{~K}$ to $20 \mathrm{~K}$ in $2 \mathrm{~K}$ steps, labelled for $20 \mathrm{~K}$

$20 \mathrm{~K}$ to $50 \mathrm{~K}$ in $5 \mathrm{~K}$ steps, labelled for $50 \mathrm{~K}$

above $50 \mathrm{~K}$ in $50 \mathrm{~K}$ steps, labelled for $200 \mathrm{~K}, 300 \mathrm{~K}$ and $400 \mathrm{~K}$.

Figure 4 shows the "diffuse component" for the same fields as Fig. 3, but equatorial coordinates are shown for Epoch 2000.0. These intensities include the all-sky background temperature of $2.8 \mathrm{~K}$. Contours are shown every $50 \mathrm{mK}$ and are labelled every $250 \mathrm{mK}$.

A list of small diameter sources was derived by fitting a single elliptical or a circular Gaussian in small area around individual sources. Only sources with fitted flux densities exceeding $160 \mathrm{mK} T_{B}$ (or $79 \mathrm{mJy} /$ beam) and sizes smaller than $16^{\prime}$ have been included. We have calculated an integrated flux density for all sources based on the peak flux density and the fitted size. The list of 1830 compact sources is available in electronic form at the CDS (anonymous ftp 130.79.128.5). The list is merged with that of Paper I (see description of individual columns), where 884 sources have been fitted with peak flux densities exceeding $98 \mathrm{mJy}$ or $200 \mathrm{mK} T_{B}$. Because of residual sidelobes near to CAS A we have not tried to fit sources within the area $110^{\circ} \leq l \leq 113^{\circ}$ and $-3.5 \leq b \leq-0.5$. CAS A itself is so strong that nonlinearities of the receiver make a flux determination unreliable.

\section{Concluding remarks}

With this paper the $21 \mathrm{~cm}$ Galactic plane survey project carried out with the Effelsberg 100-m telescope is completed. A similar Galactic plane survey at $11 \mathrm{~cm}$ wavelength is already available (Reich et al. 1990b; Fürst et al. 1990). Both surveys and other radio continuum surveys can be accessed in FITS-format via the World Wide Web (http://www.mpifr-bonn.mpg.de/survey.html). Details of the access and the retrieval software have been described by Fürst et al. (1997) and is also available at the given World Wide Web address.

Acknowledgements. We like to thank O. Lochner and his group for support and help with selecting observing bands free of interference. We are grateful to R. Wielebinski for his continuous support and interest in the Effelsberg continuum surveys. We thank J. Lequeux for helpful comments. 


\section{References}

Emerson D.T., Gräve R., 1988, A\&A 190, 353

Fürst E., Reich W., Reich P., Uyaniker B., Wielebinski R., 1997, Proc. of IAU 179, "New Horizons from Multi-Wavelength Sky Surveys" (in press)

Fürst E., Reich W., Reich P., Reif K., 1990, A\&AS 85, 691

Haslam C.G.T., Salter C., Stoffel H., Wilson W.E., 1982, A\&AS 47, 1
Kalberla P.M.W., Mebold U., Reich W., 1980, A\&A 82, 275 Kallas E., Reich W., 1980, A\&AS 42, 227

Reich W., 1982, A\&AS 48, 219

Reich P., Reich W., 1986, A\&AS, 63, 205

Reich W., Fürst E., Arnal E.M., 1992, A\&A 256, 214

Reich W., Reich P., Fürst E., 1990a, A\&AS 83, 539

Reich W., Fürst E., Reich P., Reif K., 1990b, A\&AS 85, 633

Schmidt A., Wongsowijoto S., 1983, Kleinheubacher Berichte 26,223 

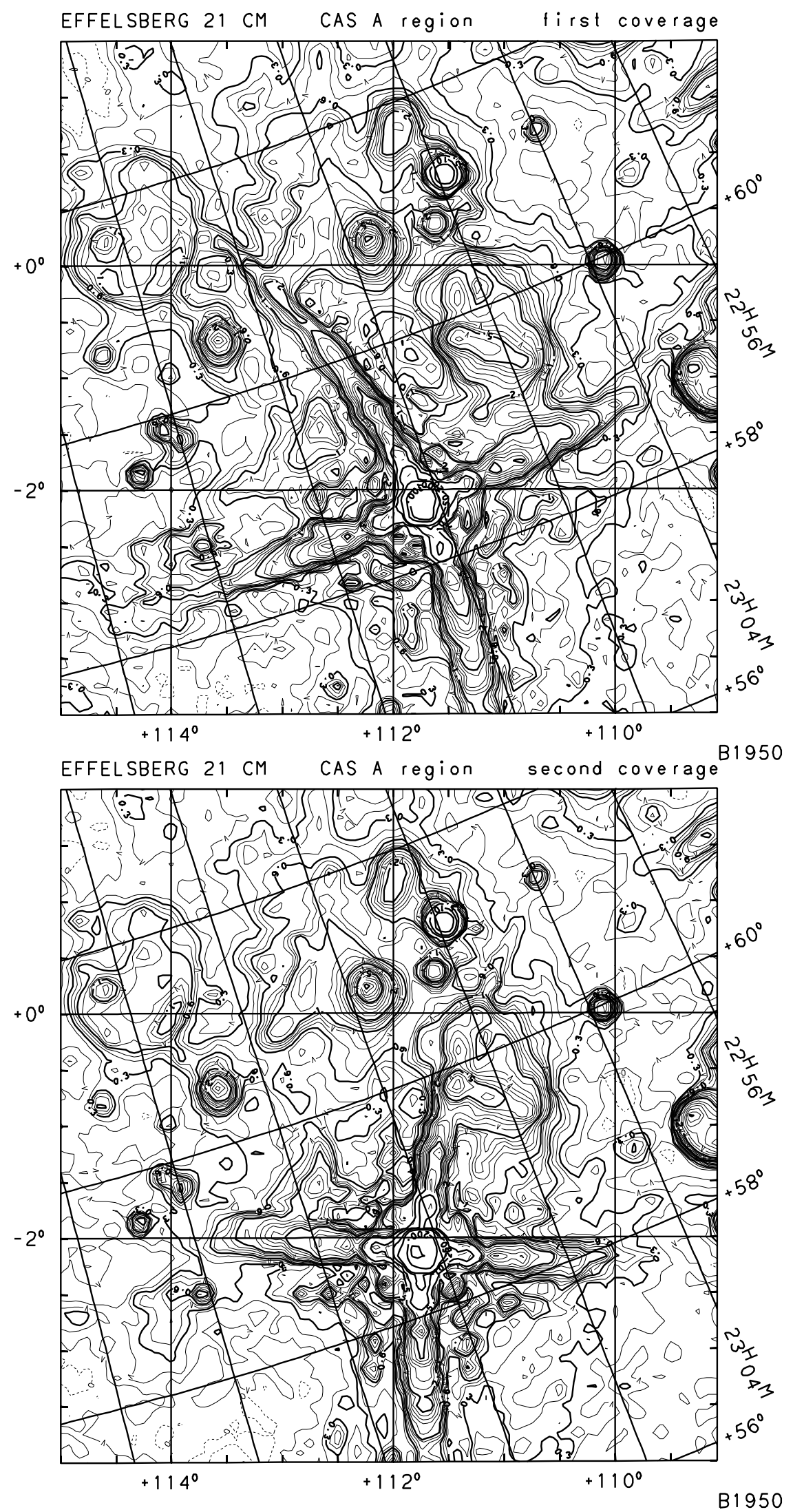

Fig. 1. a and b)Strong sidelobe distortions from CAS A. The two maps have been observed at different parallactic angles. Contour steps are the same as for Fig. 3 and listed in Sect. 5 

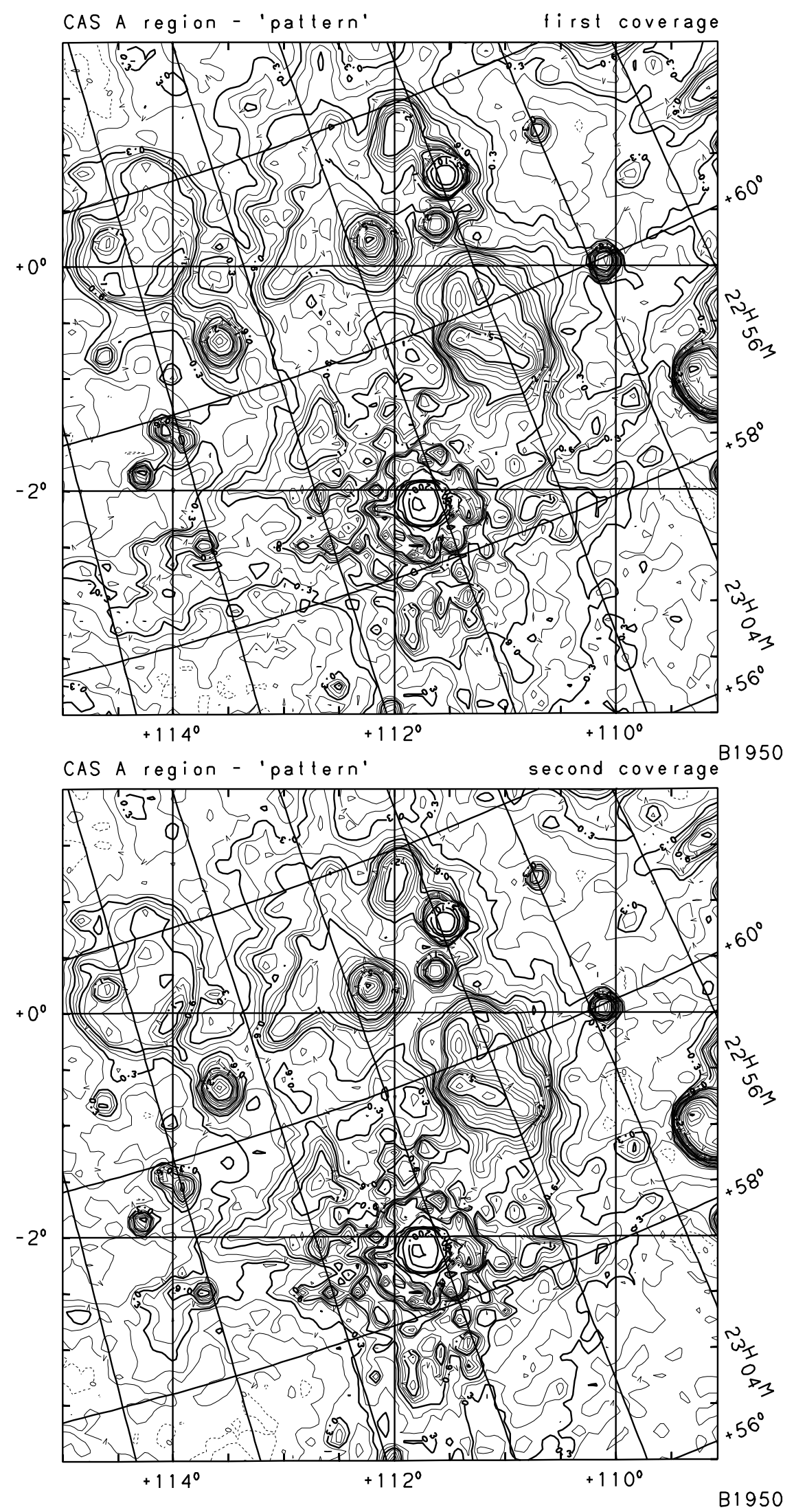

Fig. 2. a and b) Sidelobe corrected maps of the CAS A area as described in Sect. 4 


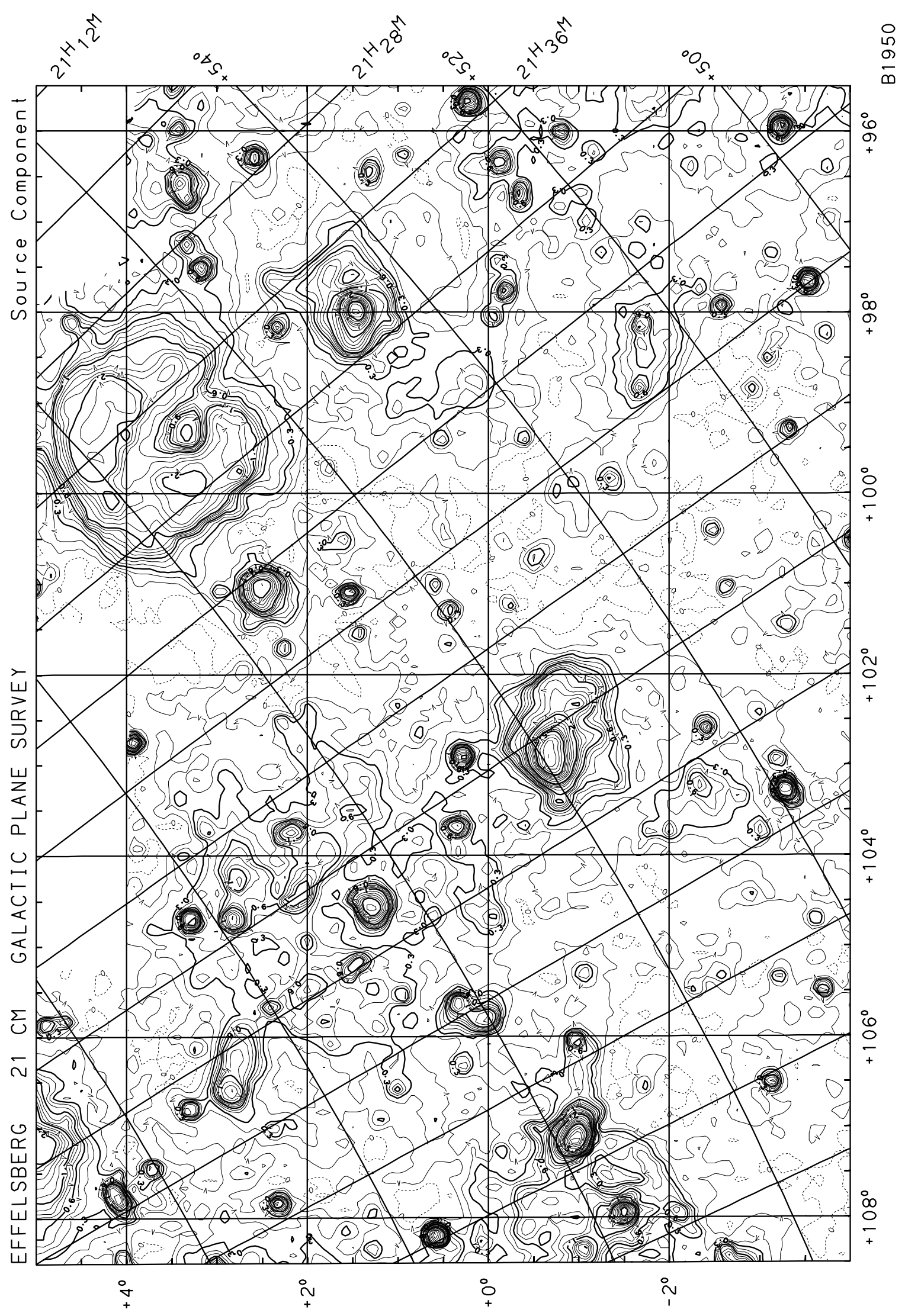

Fig. 3. a-1) The atlas of contour maps in Galactic coordinates ordered for increasing longitude showing the "source component" (see Sect. 3). Contour steps are listed in Sect. 5 


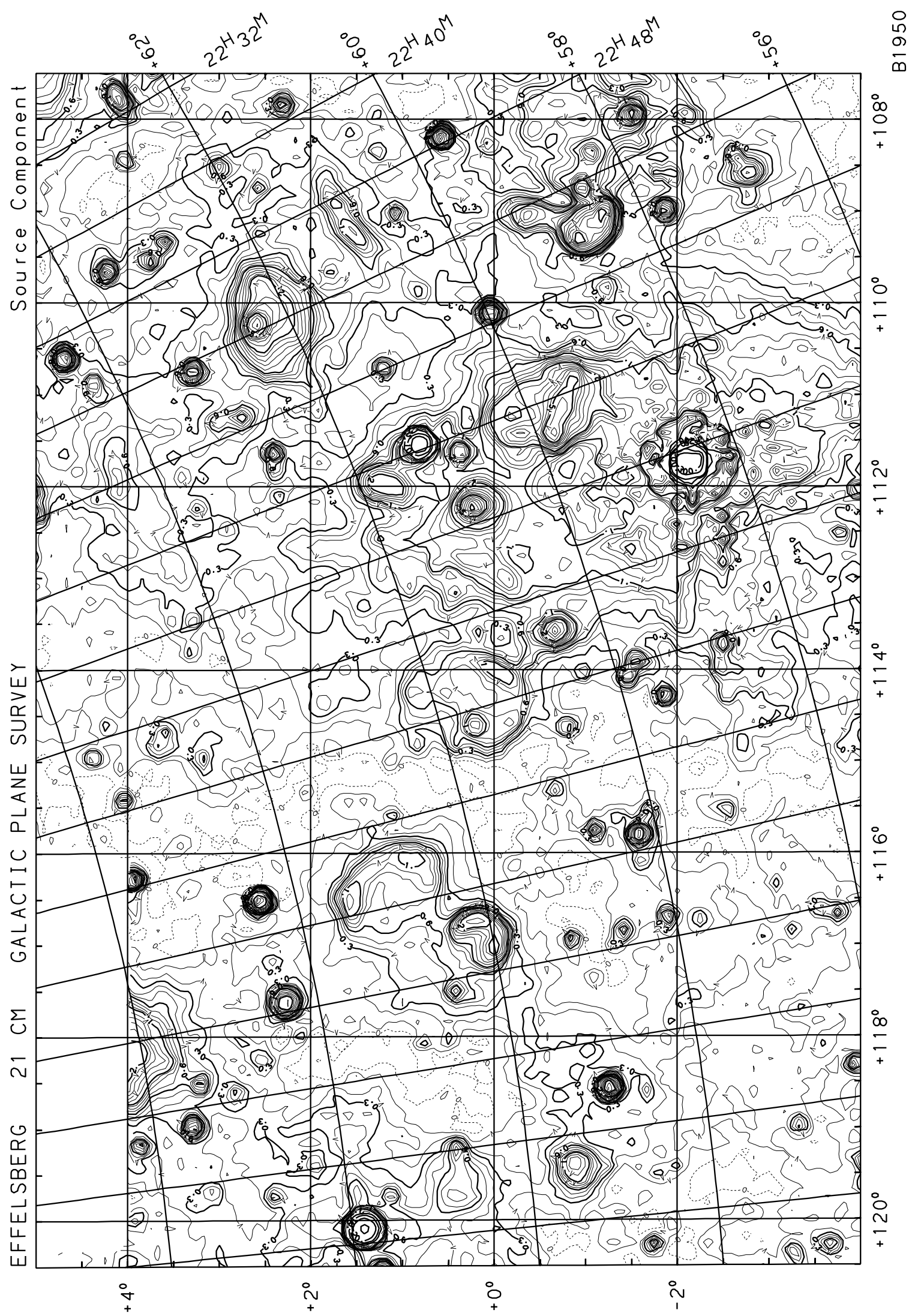

Fig. 3. b) continued 


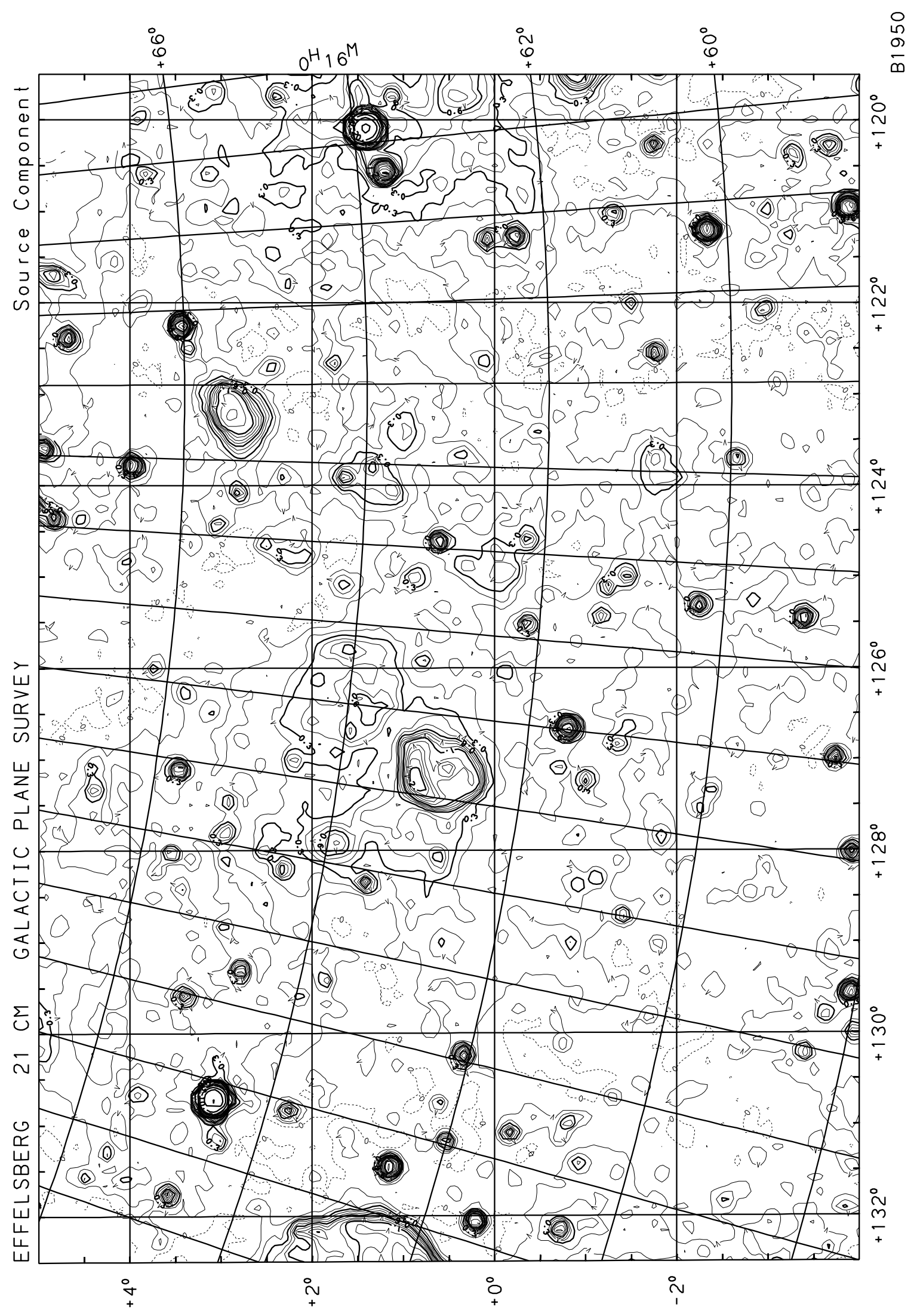

Fig. 3. c) continued 


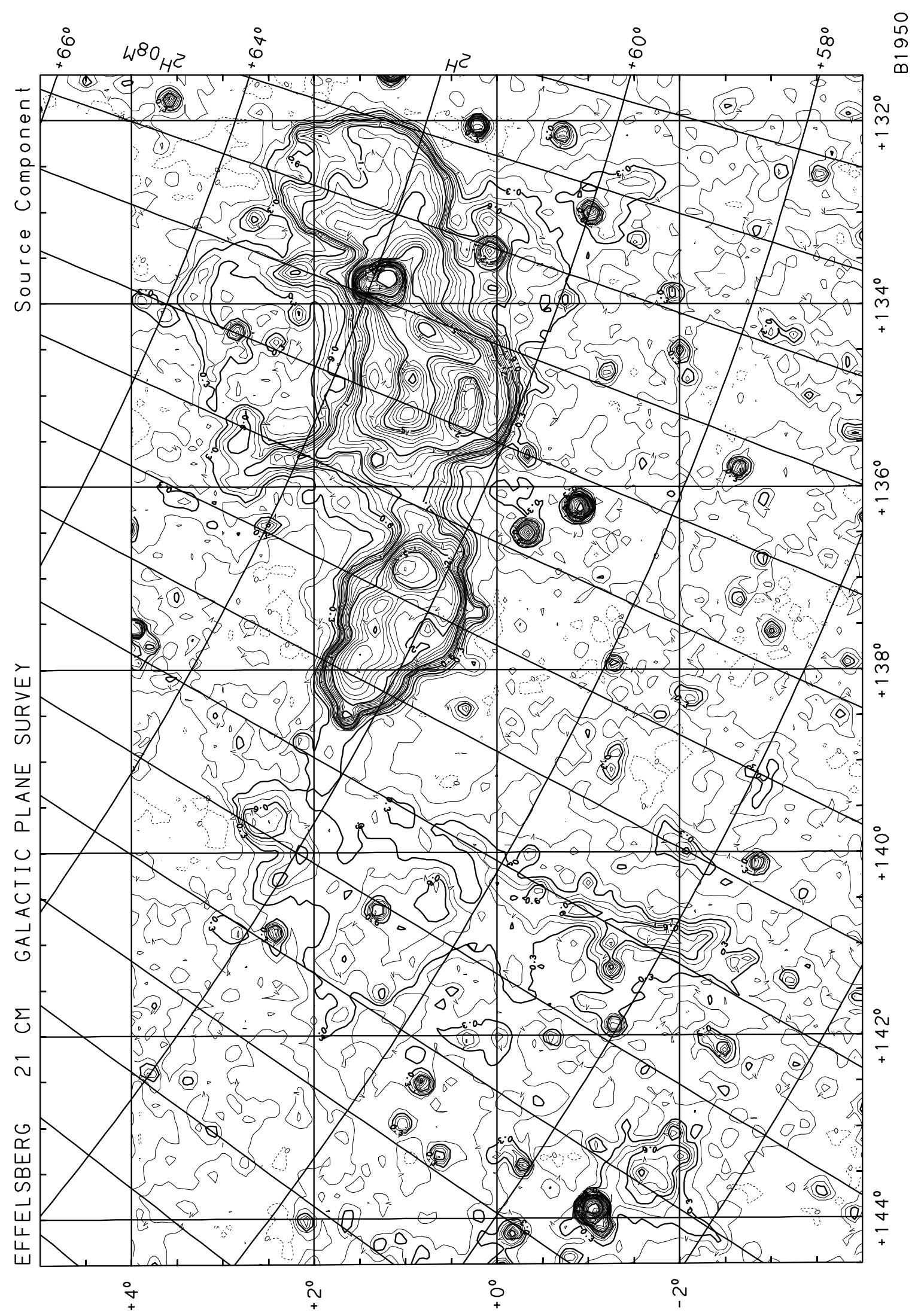

Fig. 3. d) continued 


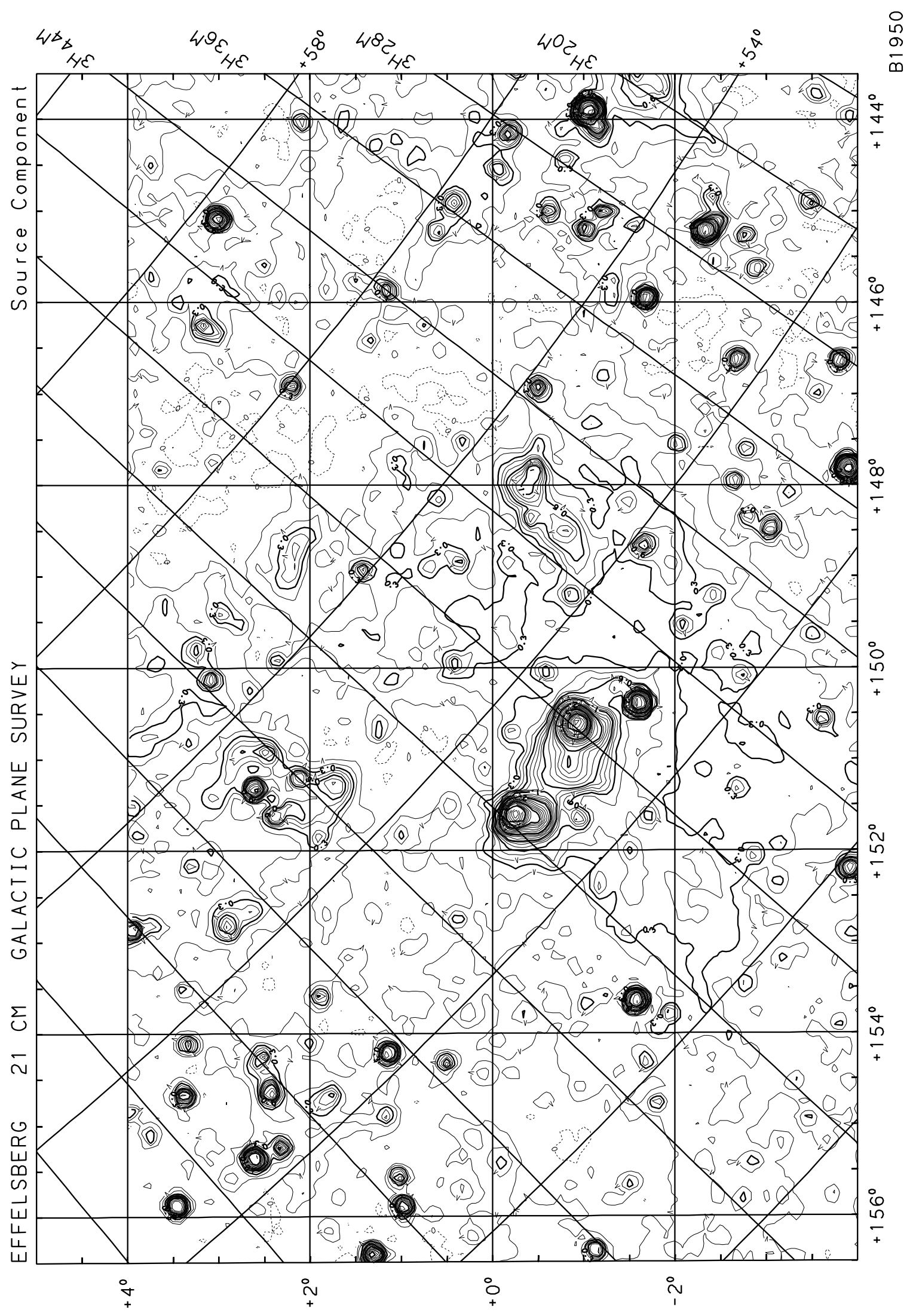

Fig. 3. e) continued 


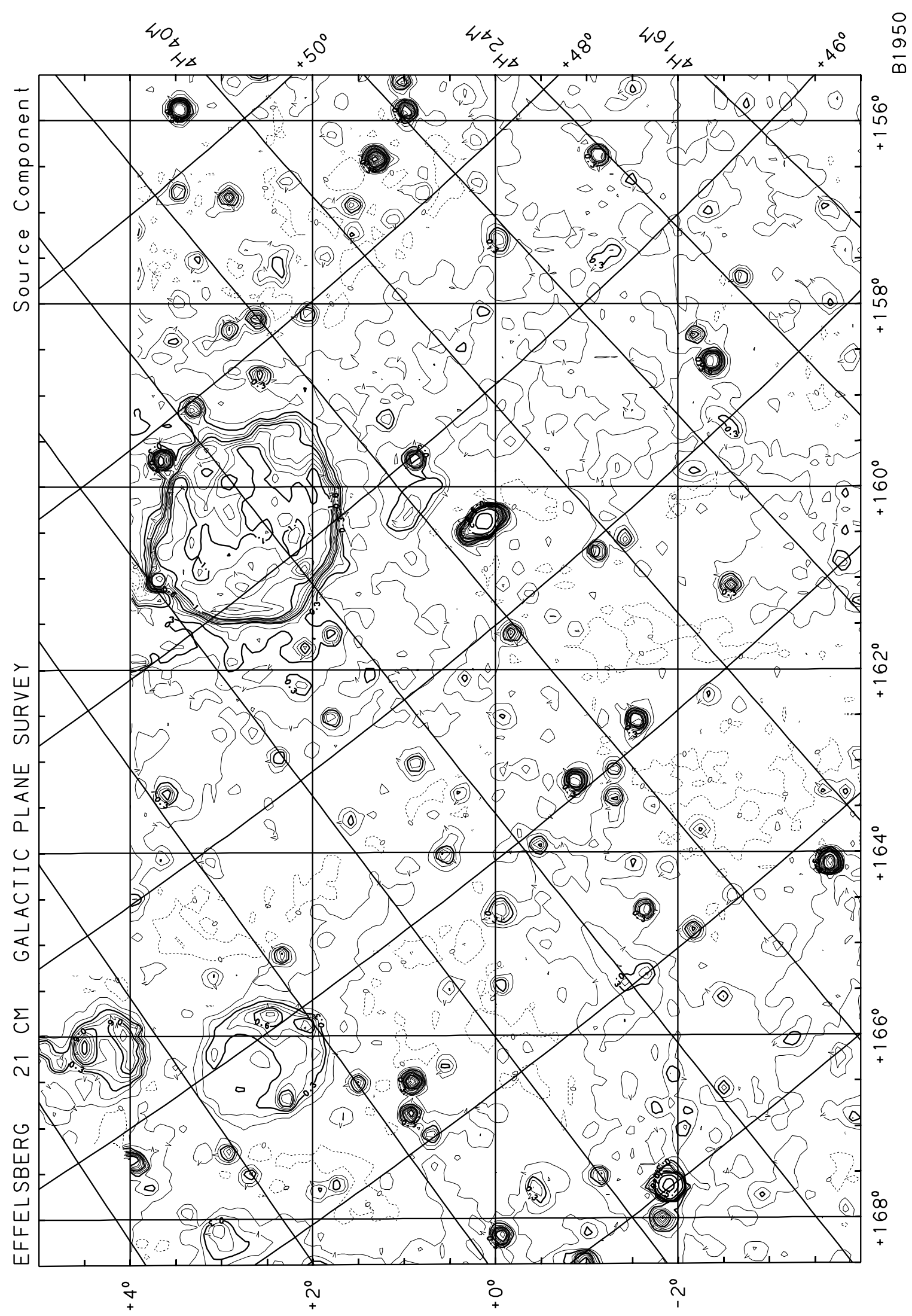

Fig. 3. f) continued 


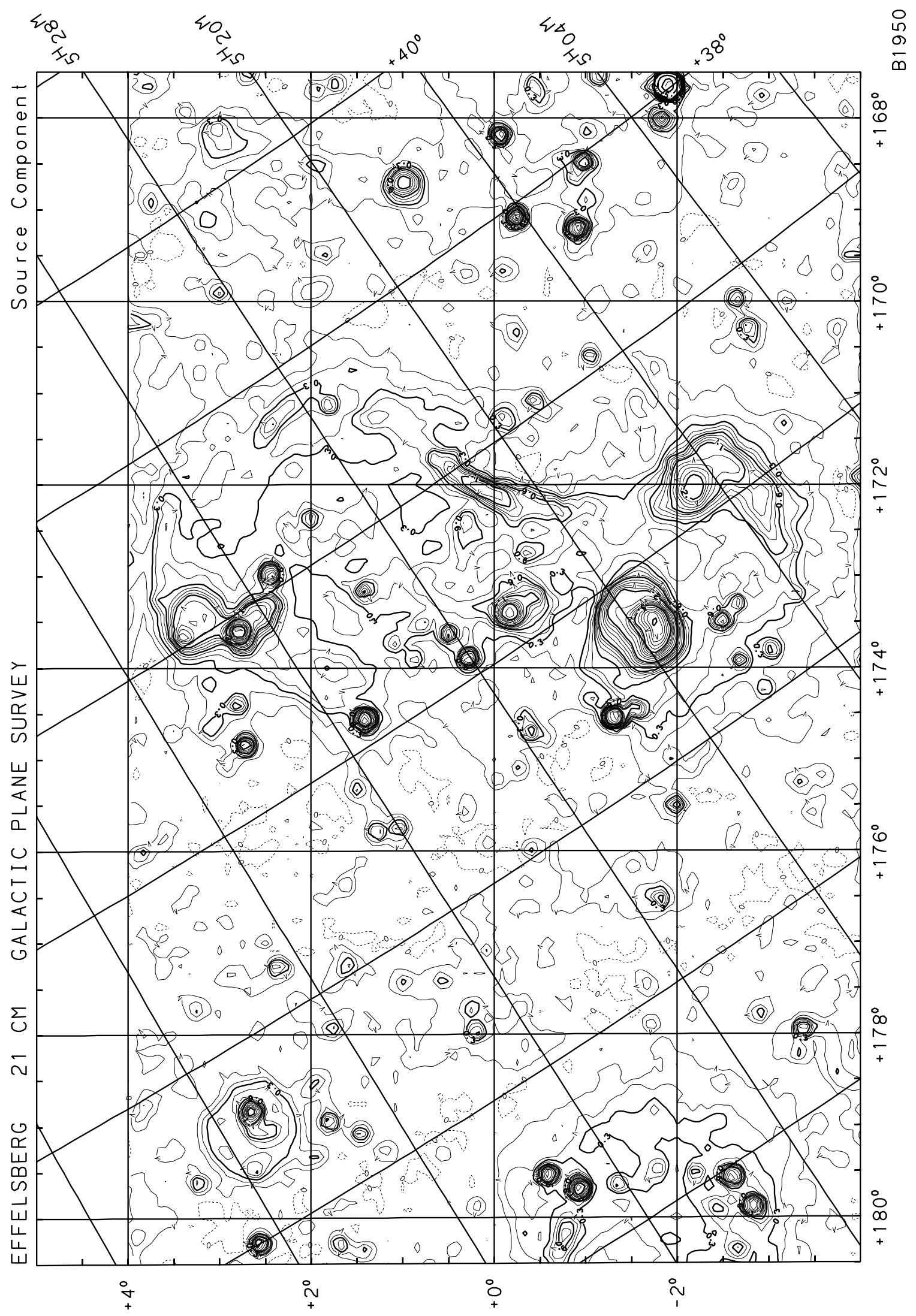

Fig. 3. g) continued 


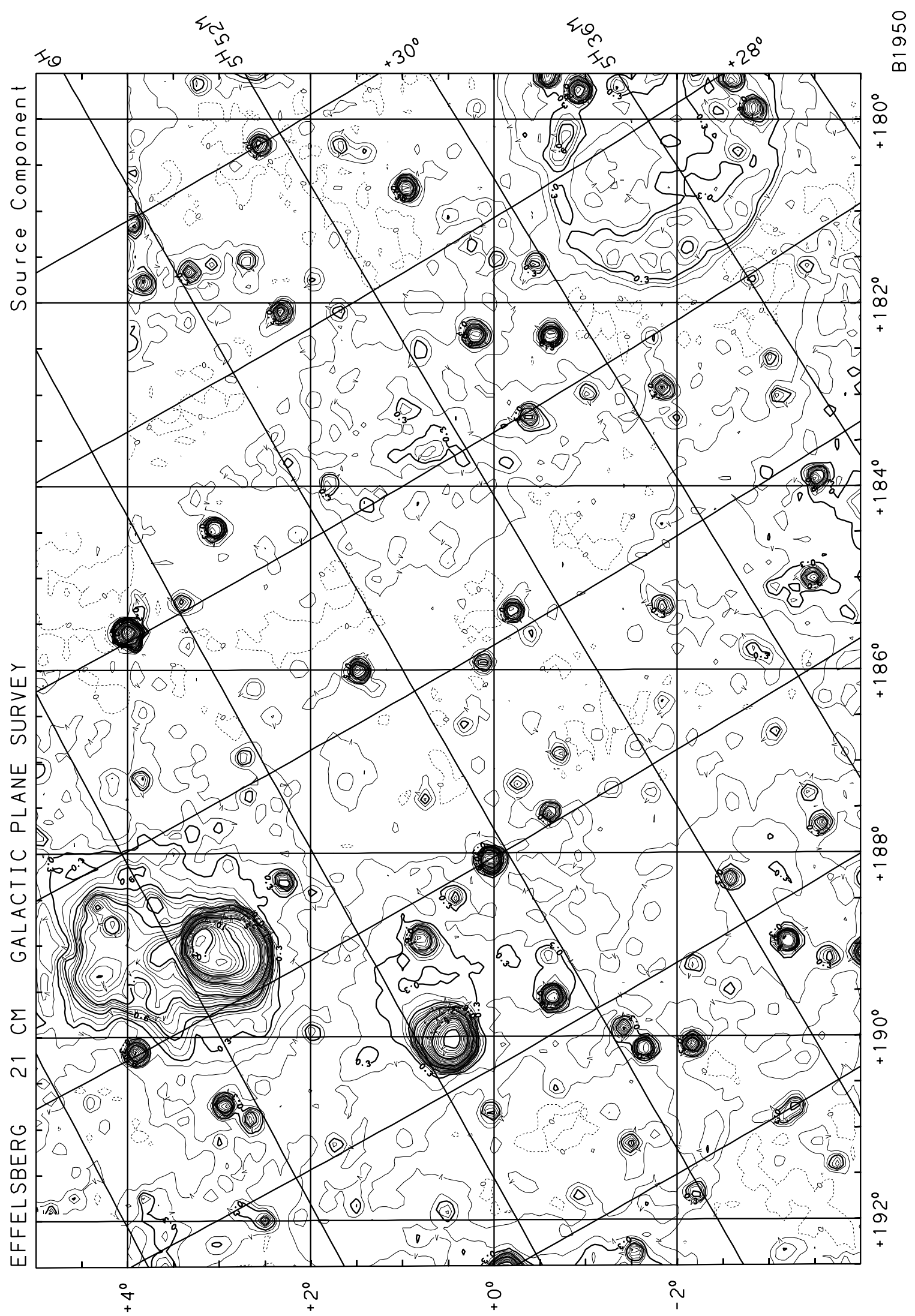

Fig. 3. h) continued 


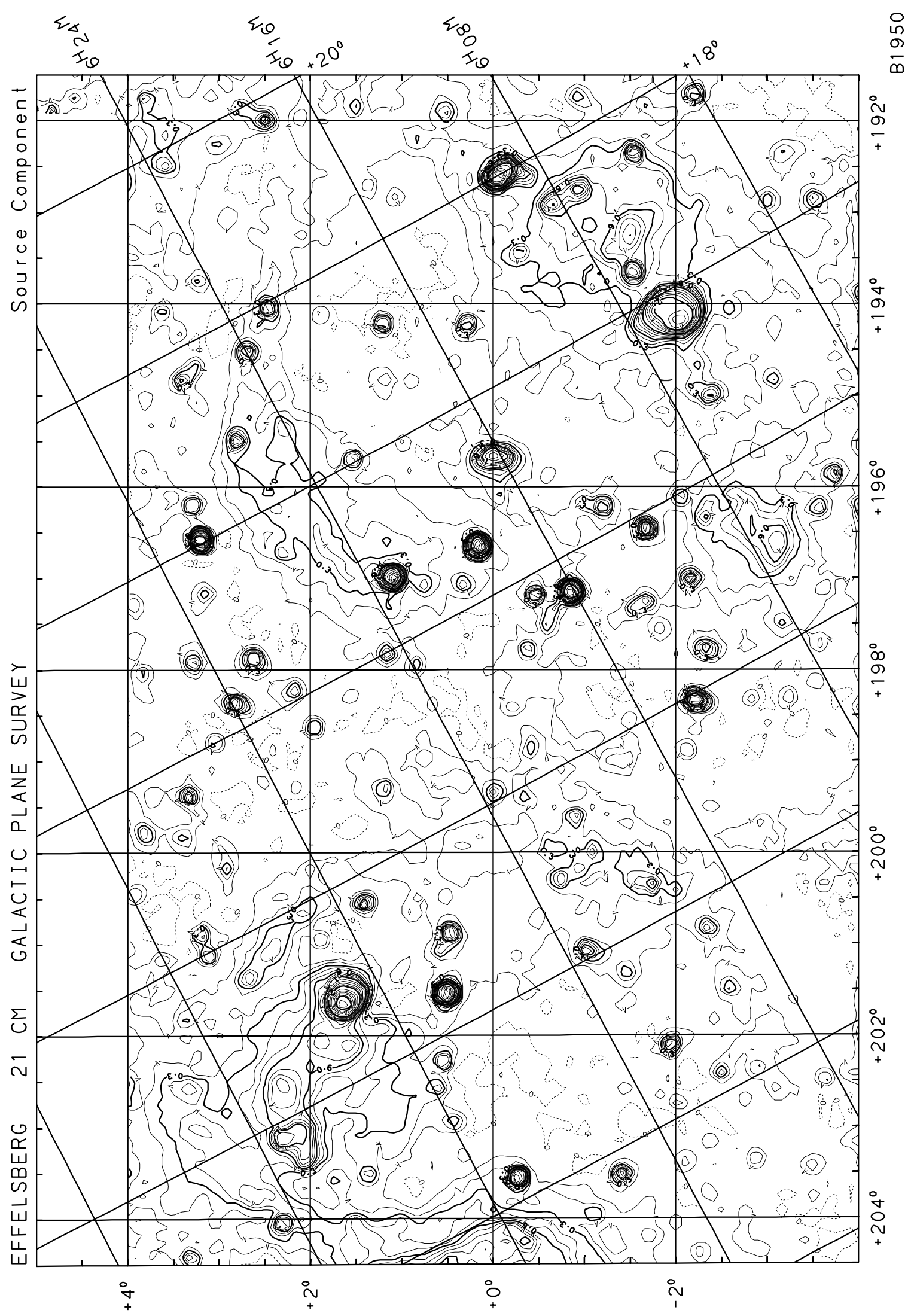

Fig. 3. i) continued 


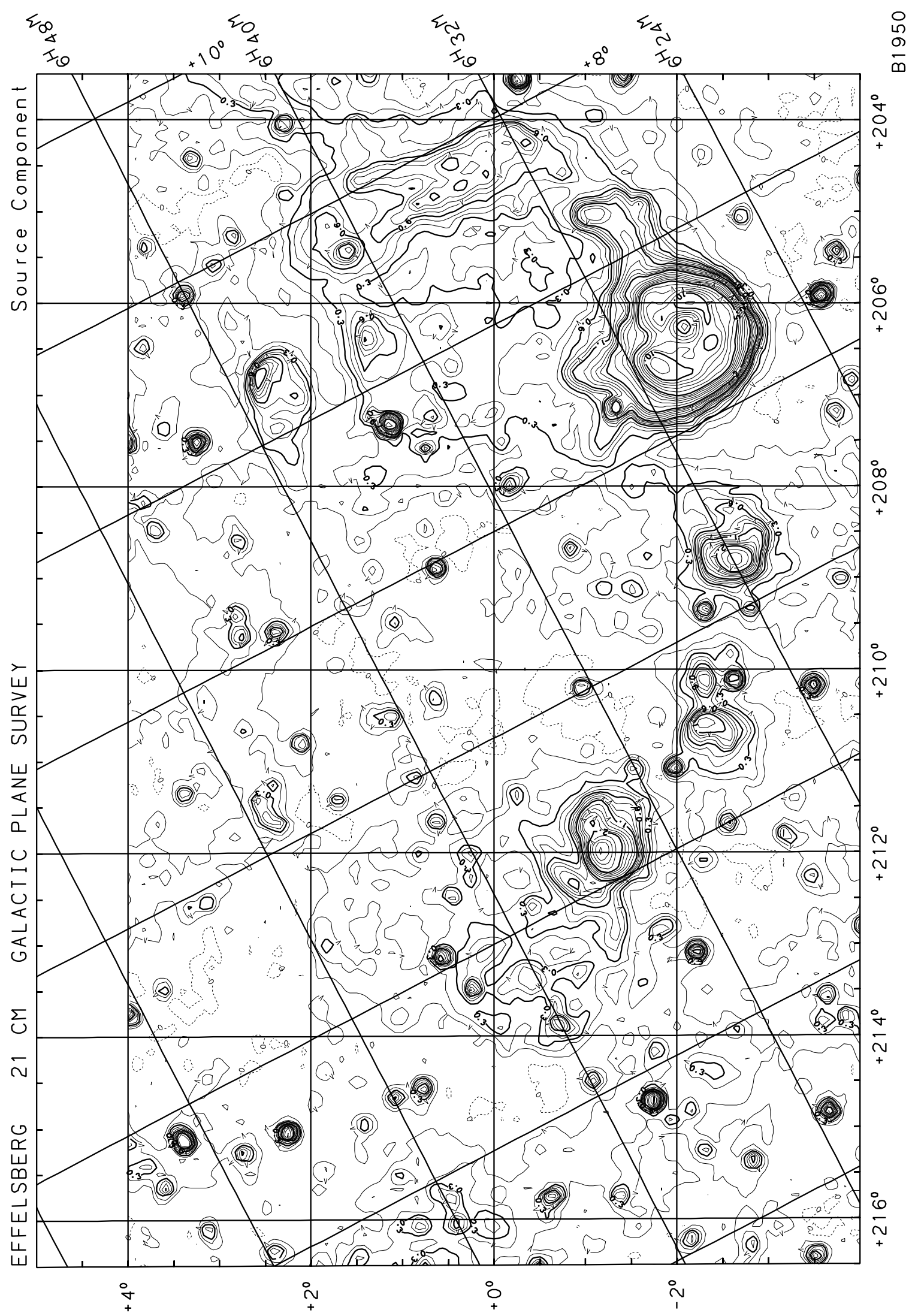

Fig. 3. j) continued 


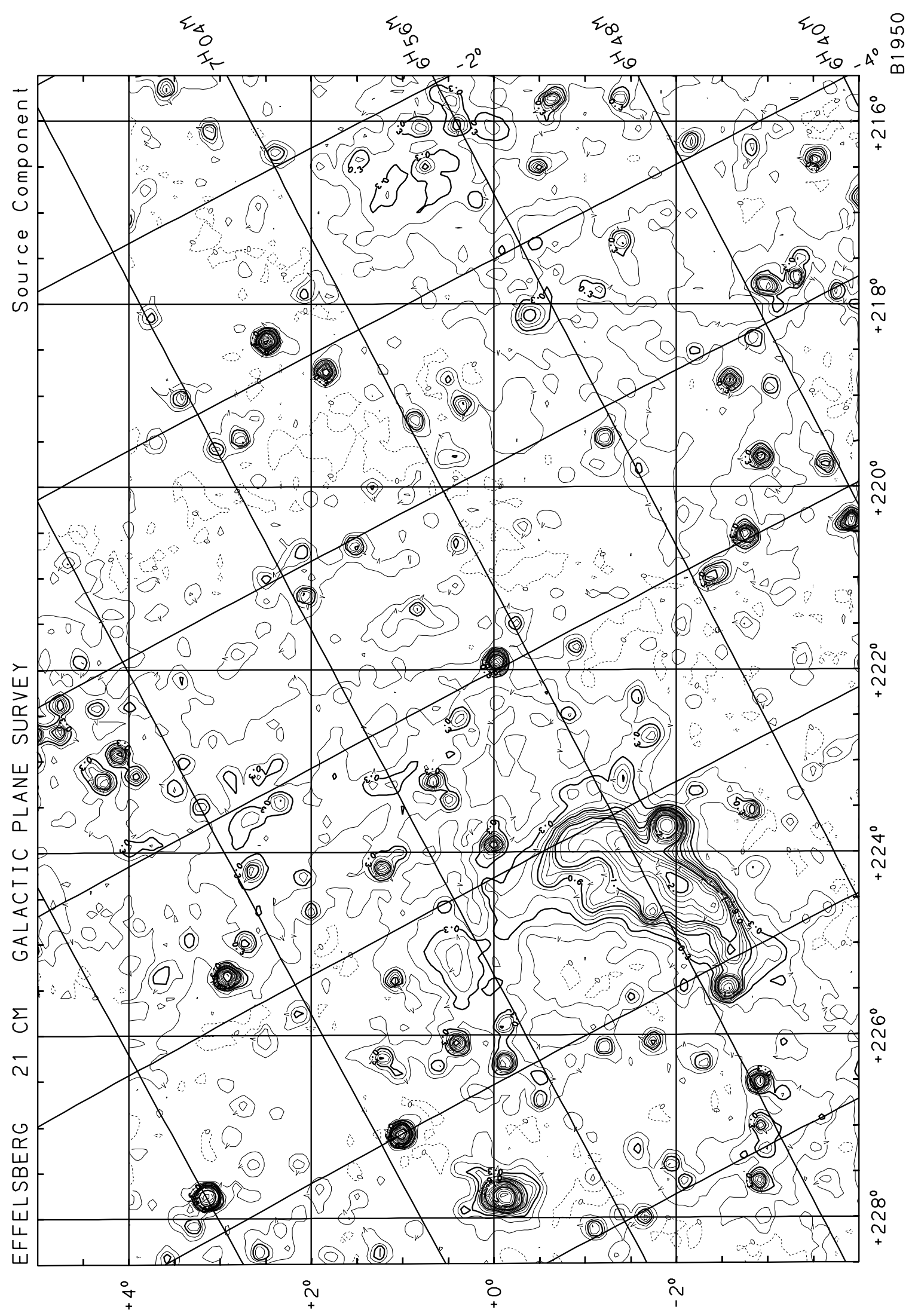

Fig. 3. k) continued 


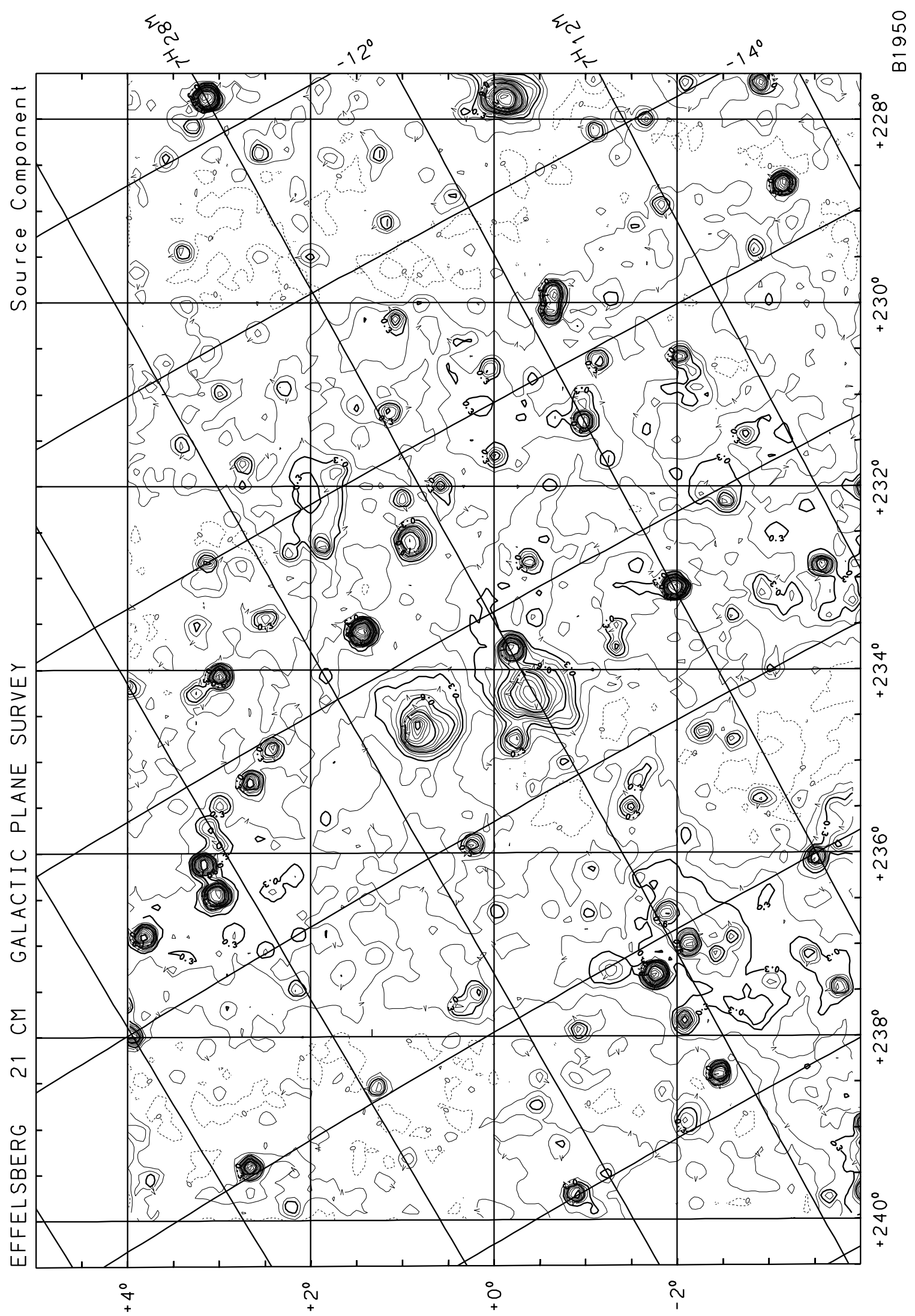

Fig. 3. 1) continued 


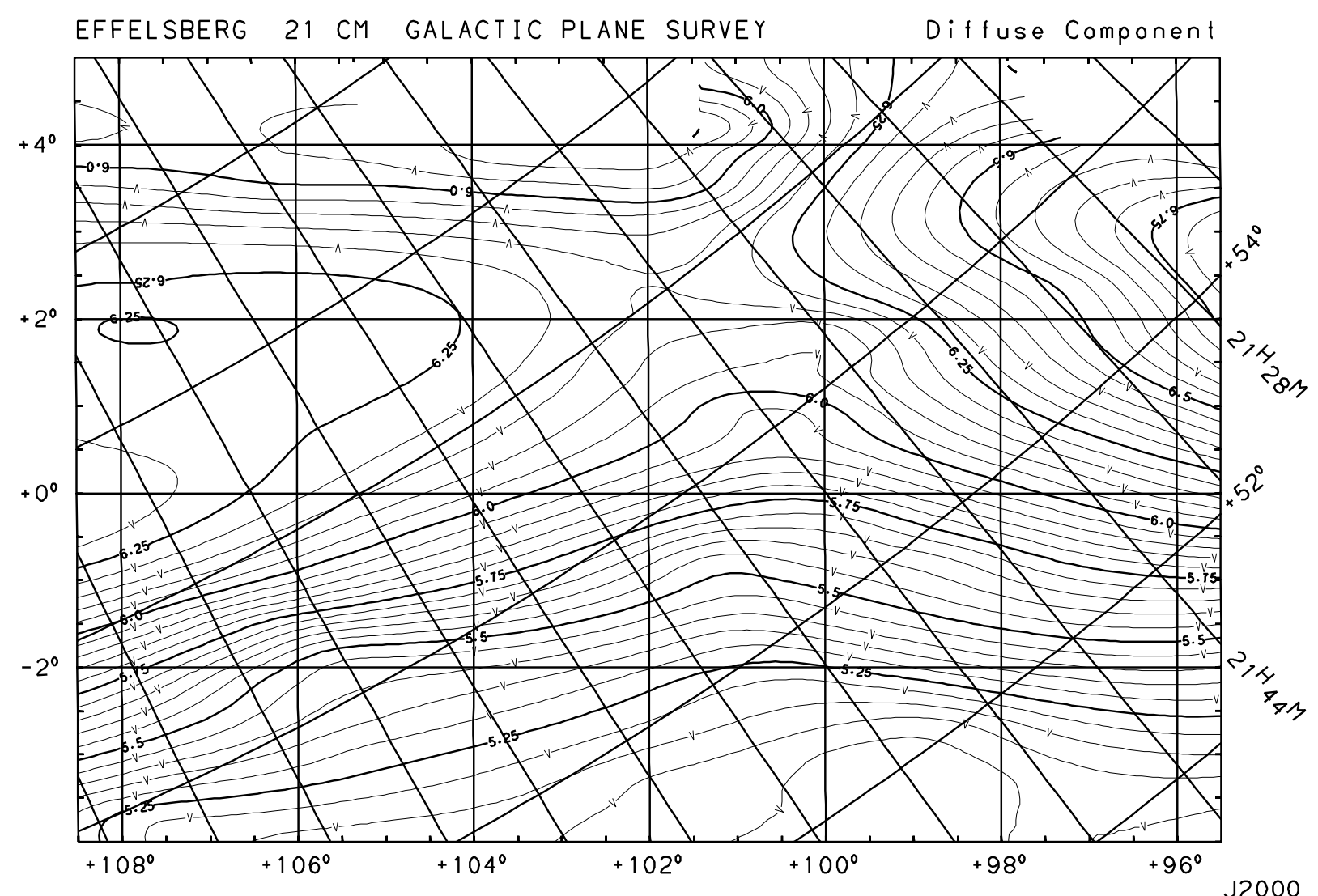

EFFELSBERG 21 CM GALACTIC PLANE SURVEY

Diffuse Component
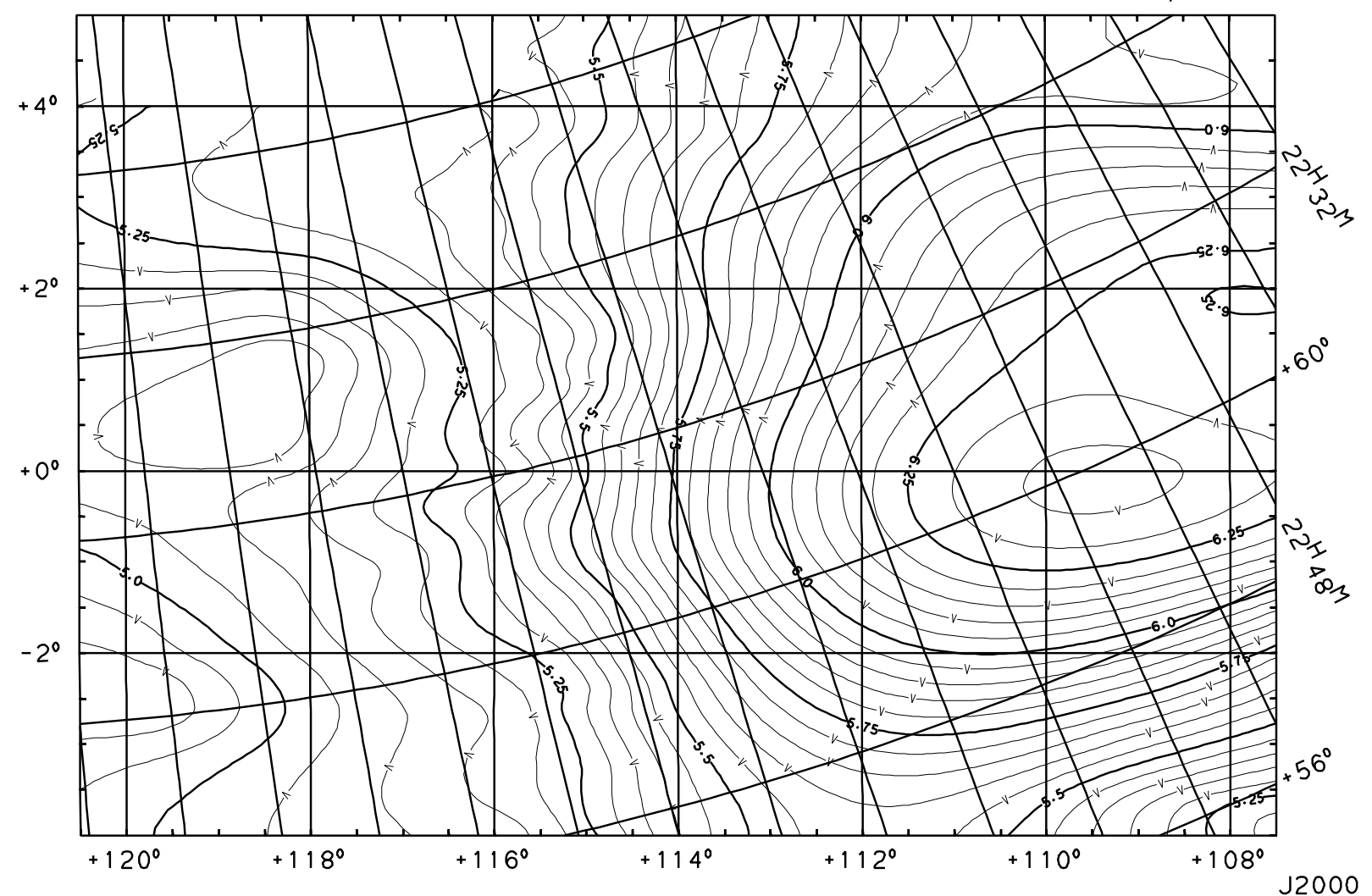

Fig. 4. a-1) As Fig. 3, but showing the "diffuse component" (see Sect. 3). Contour steps are listed in Sect. 5 

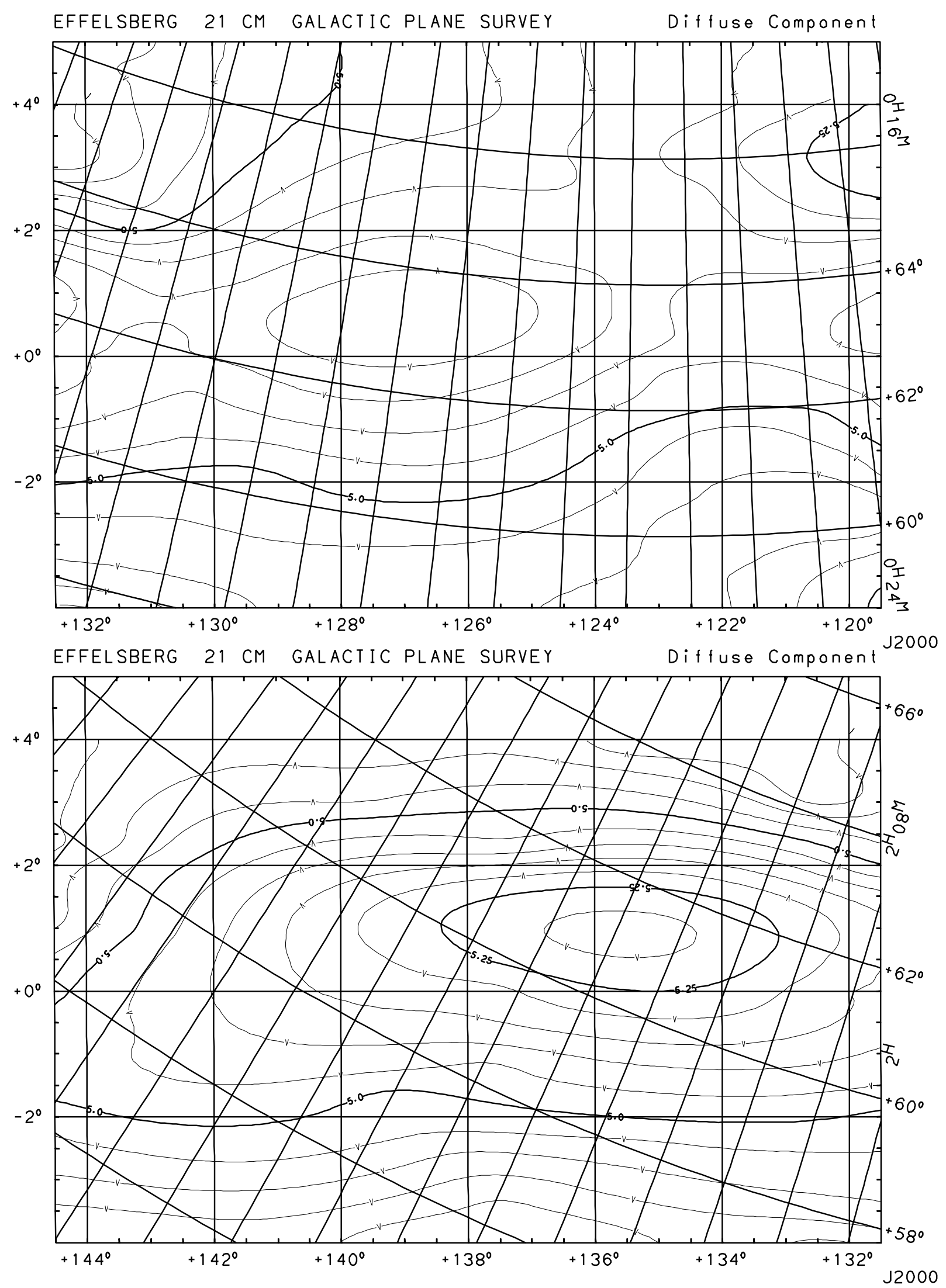

Fig. 4. c-d) continued 

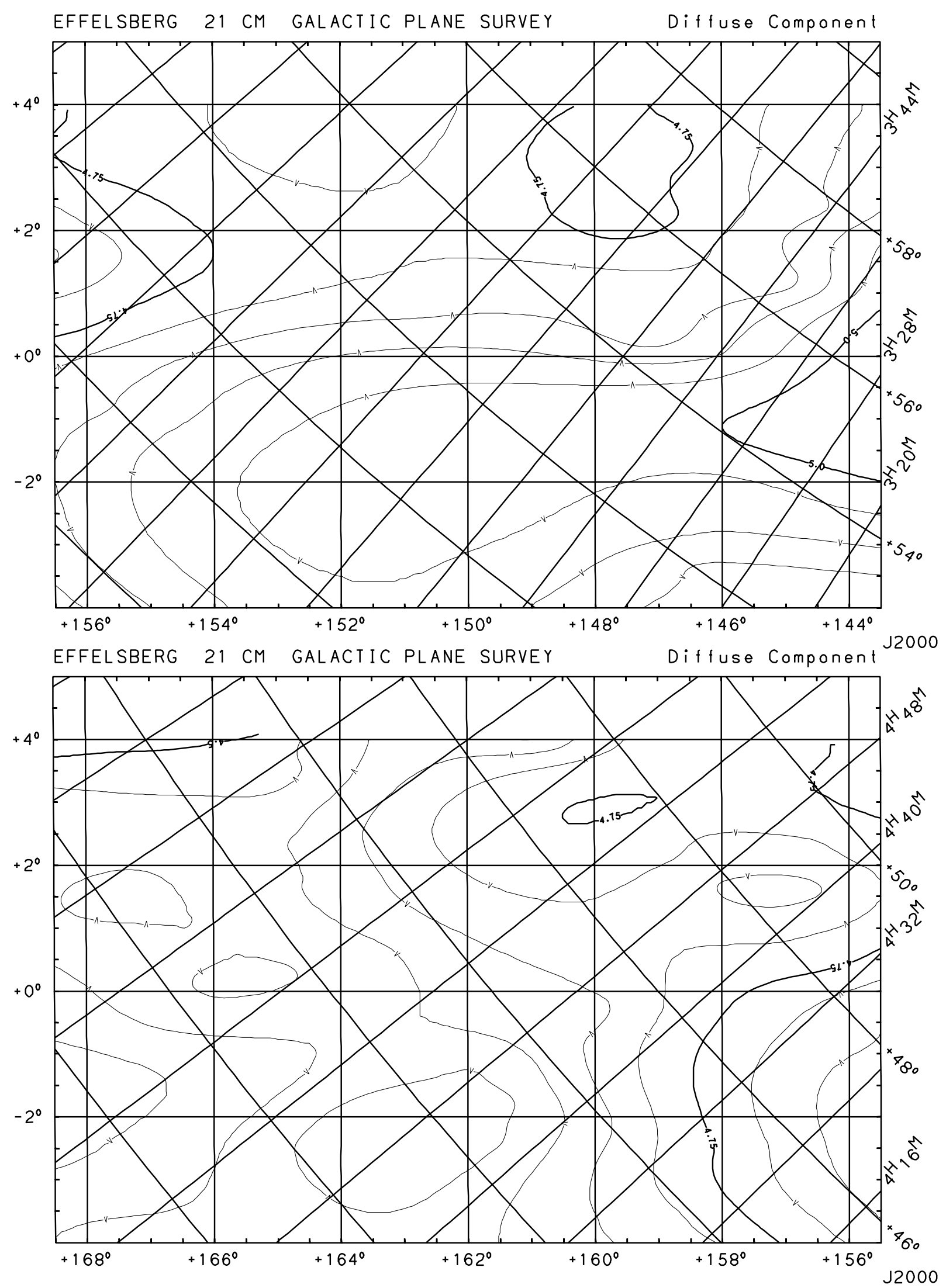

Fig. 4. e-f) continued 

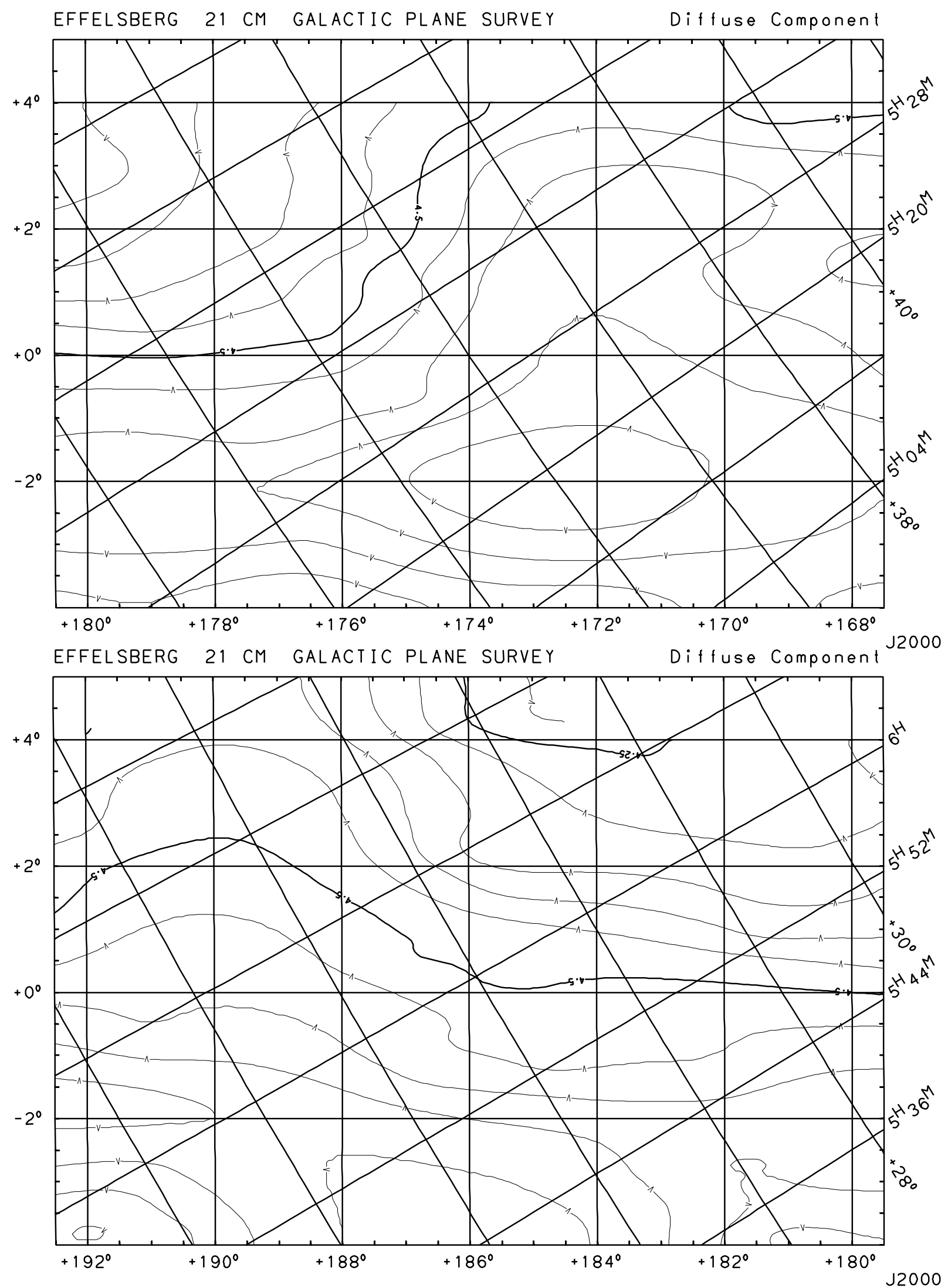

Fig. 4. g-h) continued 

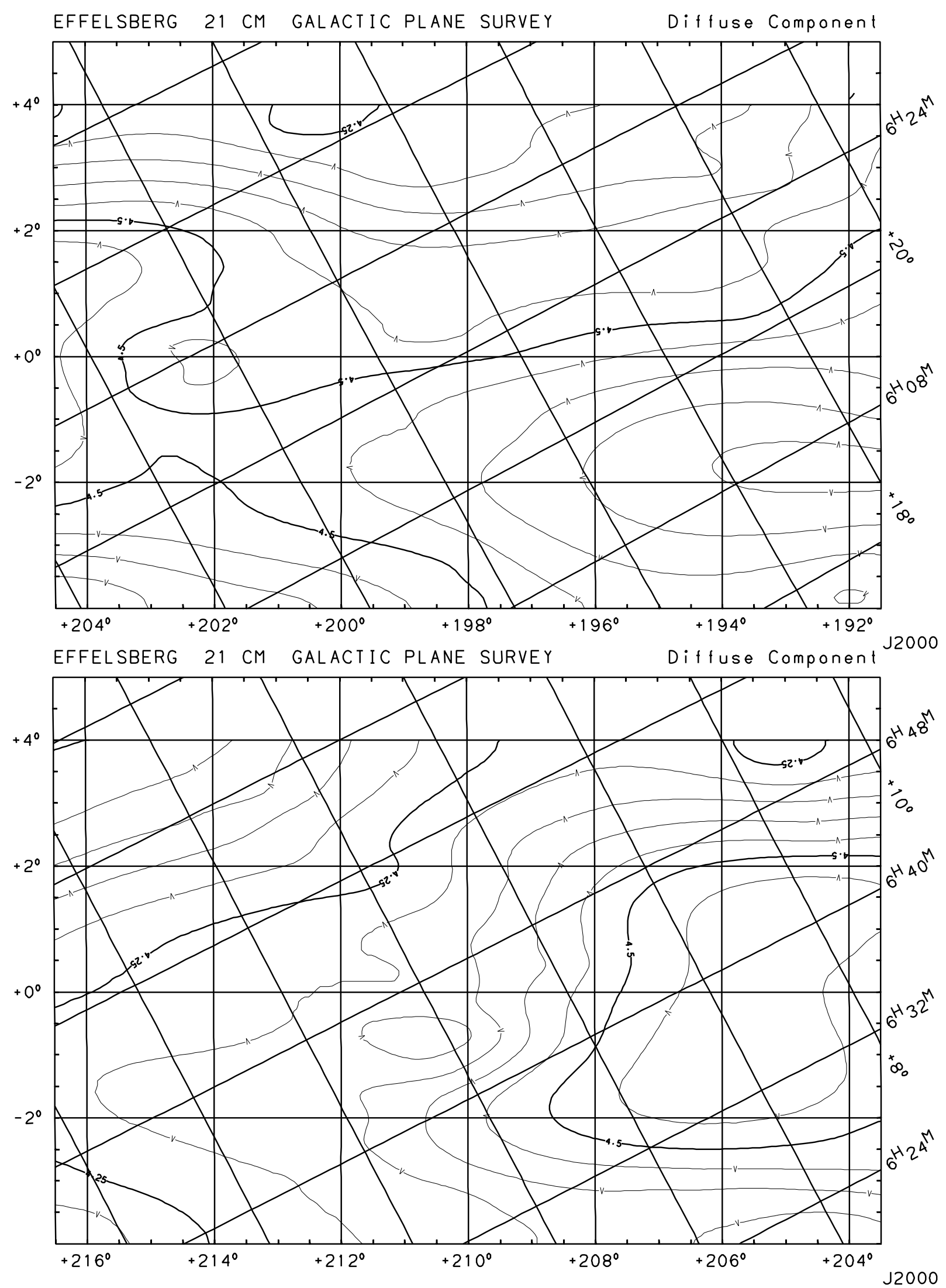

Fig. 4. i-j) continued 

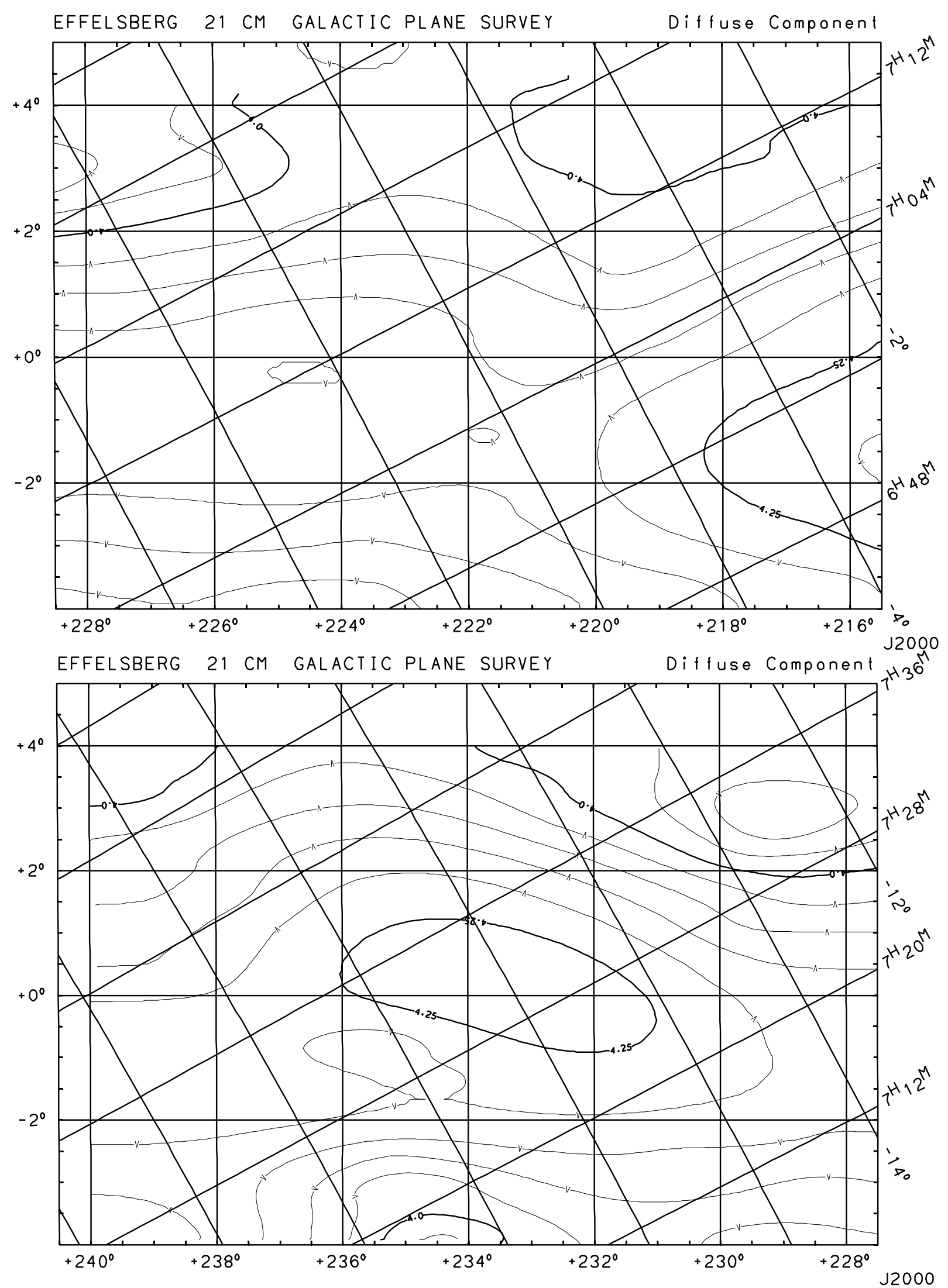

Fig. 4. k-l) continued 